
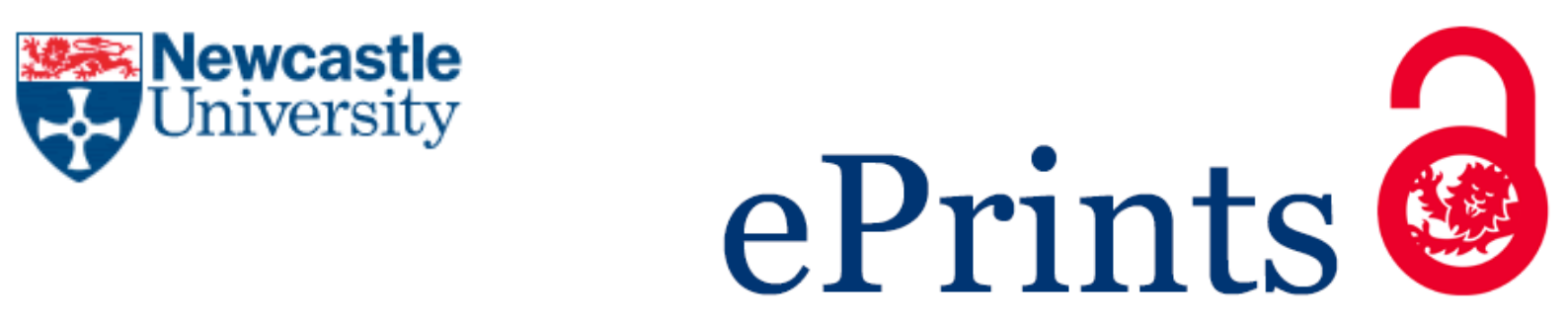

Wesnes KA, Brooker H, Watson AW, Bal W, Okello E.

Effects of the Red Bull Energy Drink on cognitive function and mood in

healthy young volunteers.

Journal of Psychopharmacology (2016)

DOI: https://doi.org/10.1177/0269881116681459

\title{
Copyright:
}

This is the authors' accepted manuscript of an article that has been published in its final definitive form by Sage Publications, on behalf of the British Association for Psychopharmacology, 2016.

DOI link to article:

https://doi.org/10.1177/0269881116681459

Date deposited:

$09 / 11 / 2016$

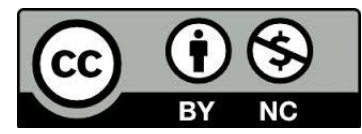

This work is licensed under a Creative Commons Attribution-NonCommercial 3.0 Unported License 
Journal of

Psychopharmacology

\section{Effects of the Red BulI $($ Energy Drink on cognitive function and mood in healthy young volunteers}

\begin{tabular}{|c|c|}
\hline Journal: & Journal of Psychopharmacology \\
\hline Manuscript ID & JOP-2016-2895.R2 \\
\hline Manuscript Type: & Original Paper \\
\hline Date Submitted by the Author: & $21-$ Oct-2016 \\
\hline Complete List of Authors: & $\begin{array}{l}\text { Wesnes, Keith; Wesnes Cognition Ltd, Little Paddock } \\
\text { Brooker, Helen; Wesnes Cognition Ltd, Research } \\
\text { Watson, Anthony; Newcastle University, NU-Food Research Facility } \\
\text { Bal, Wendy; Newcastle University, Human Nutrition Research Centre } \\
\text { Okello, Edward; Newcastle University, Medicinal Plant Research Group/ } \\
\text { Human Nutrition Research centre }\end{array}$ \\
\hline \multirow[t]{2}{*}{$\begin{array}{r}\text { Please list at least } 3 \text { keywords } \\
\text { which relate to your } \\
\text { manuscript:: }\end{array}$} & Red Bull, Memory, Computerised Cognitive Testing \\
\hline & 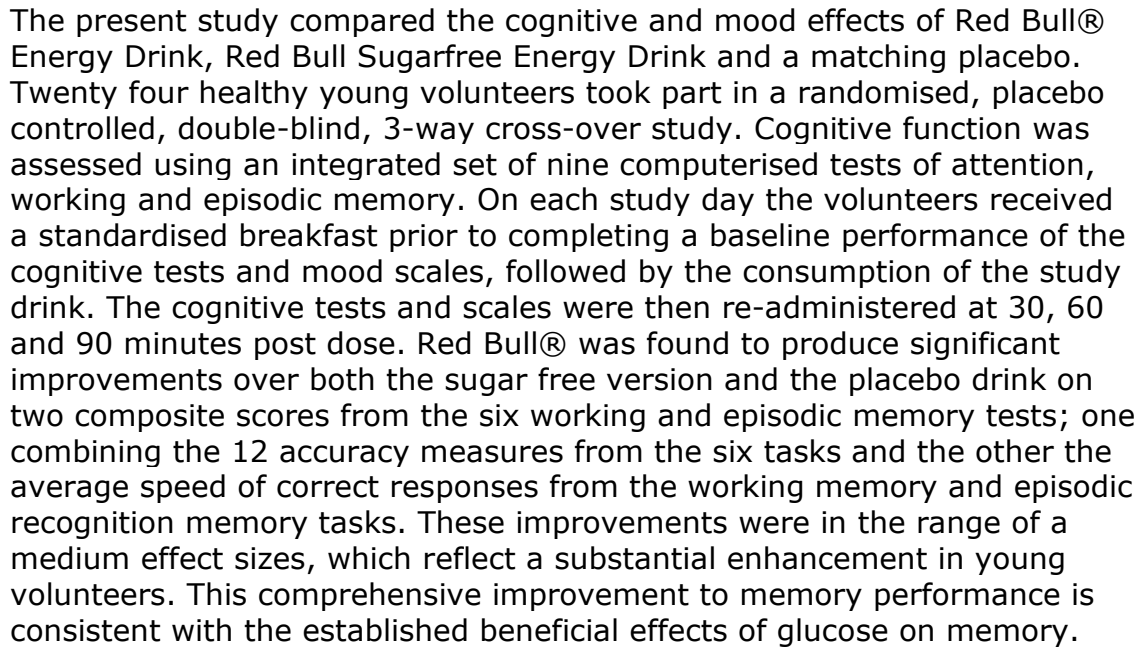 \\
\hline
\end{tabular}

\section{SCHOLARONE" \\ Manuscripts}


Red Bull and cognitive function

\title{
Effects of the Red BulI® Energy Drink on cognitive function and mood in healthy young volunteers
}

\author{
Keith A. Wesnes * \\ Wesnes Cognition Ltd, Streatley on Thames, UK \\ Medical School, University of Exeter-Medical School, Exeter, UK \\ Department of Psychology, Northumbria University, Newcastle, UK \\ Centre for Human Psychopharmacology, Swinburne University, Melbourne, Australia \\ Medicinal Plant Research Group, Newcastle University, UK \\ Helen Brooker \\ Wesnes Cognition Ltd, Streatley on Thames, UK \\ Anthony W. Watson
}

NU-Food Research Facility, Human Nutrition Research Centre, Newcastle University, UK Wendy Bal

NU-Food Research Facility, Human Nutrition Research Centre, Newcastle University, UK Ed Okello

Medicinal Plant Research Group/ Human Nutrition Research centre, Newcastle University, UK 
Red Bull and cognitive function

* Corresponding Author

Keith A. Wesnes

Little Paddock,

Streatley Hill,

Streatley on Thames,

RG8 9RD, UK

Email: keith@wesnes.com

Tel +44 (0) 7768960339

\section{DISCLOSURES}

The CogTrack ${ }^{\mathrm{TM}}$ System is proprietary to Wesnes Cognition Ltd (www.wesnes.com) who provided financial support for the conduct of the study and the preparation of this paper. Keith Wesnes owns Wesnes Cognition Ltd and consults for various companies involved in clinical trials.

\section{Acknowledgements}

We acknowledge that the School of Agriculture, Food and Rural Development, Newcastle University for covered staffing costs and costs associated with the use of the NU-Food Research Facility. Wesnes Cognition Ltd supplied the costs for consumables as well as the volunteers' honoraria. We thank the developer of the Caffeine Intake Tool, Katharina Schlegel, Fakultät Life Sciences, Hochschule Albstadt-Sigmaringen, Sigmaringen, for permission to use the instrument. Finally, we thank the very diligent reviewers whose close 
Red Bull and cognitive function

scrutiny and highly constructive criticism of the first version of the manuscript has resulted in this much improved version.

2

3

4

5

6

7

8

9

10

11

12

13

14

15

16

17

18

19

20

21

22

23

24

25

26

27

28

29

30

31

32

33

34

35

36

37

38

39

40

41

42

43

44

45

46

47

48

49

50

51

52

53

54

55

56

57

58

59

60

http://mc.manuscriptcentral.com/jop 
Red Bull and cognitive function

\begin{abstract}
The present study compared the cognitive and mood effects of two commercially available products, Red Bull囚 Energy Drink $250 \mathrm{ml}$ and Red Bull Sugarfree Energy Drink $250 \mathrm{ml}$, together with a matching placebo $250 \mathrm{ml}$. Twenty four healthy young volunteers took part in a randomised, placebo controlled, double-blind, 3-way cross-over study. Cognitive function was assessed using an integrated set of nine computerised tests of attention, working and episodic memory. On each study day the volunteers received a standardised breakfast prior to completing a baseline performance of the cognitive tests and mood scales, followed by the consumption of the study drink. The cognitive tests and scales were then readministered at 30,60 and 90 minutes post dose. Red Bull@ was found to produce significant improvements over both the sugar free version and the placebo drink on two composite scores from the six working and episodic memory tests; one combining the 12 accuracy measures from the six tasks and the other the average speed of correct responses from the working memory and episodic recognition memory tasks. These improvements were in the range of a medium effect sizes, which reflect a substantial enhancement in young volunteers. This comprehensive improvement to memory performance is consistent with the established beneficial effects of glucose on memory.
\end{abstract}

\title{
KEY WORDS
}

Red Bull@, Red Bull Sugarfree, CogTrack ${ }^{\mathrm{TM}}$, Computerised Cognitive Testing, Attention, Memory 
Red Bull and cognitive function

\section{INTRODUCTION}

As Ishak et al. (2012) wrote: "Energy drinks are a group of beverages used by consumers to provide an extra boost in energy, promote wakefulness, maintain alertness, and provide cognitive and mood enhancement" (Page 25). Of the various commercially available products, the Red Bull囚 (RB) Energy Drink has for many years been by far the most widely sold energy drink or shot worldwide (www.caffeineinformer.com/ the-15-top-energy-drinkbrands). It has also been the most widely researched of the various products available. Red Bull in the original serving size of $250 \mathrm{ml}$ contains caffeine $80 \mathrm{mg}$, taurine $1000 \mathrm{mg}$, B-group vitamins (B3, B5, B6, B12) and glucose $27 \mathrm{~g}$.

The ingredients of RB, particularly caffeine and glucose, would be expected to produce positive effects on various aspects of cognitive function, and some evidence of such effects has previously been detected. Alford et al. (2001) studied the effect of RB $250 \mathrm{ml}$ in 12 healthy volunteers (aged 18 to 21 years). Compared to a dummy energy drink of quinine flavoured carbonated water with no added sugar, 30 minutes after consumption, immediate word recall performance was significantly improved as was performance on a number cancelation task. Gendle et al. (2009) compared the effects of RB $250 \mathrm{ml}$, RB Sugarfree and placebo in 34 young volunteers (aged 18 to 22 years) on the performance of the 14 minute computerised Connors Continuous Performance Test II, which began 30 minutes after consumption. A parallel group design was used, each group having one of the RB products, with a cross-over to the placebo product. There were no statistically significant differences compared with placebo on any of the four accuracy and speed measures from the test. Kammerer et al. (2014) compared RB 250 ml, a placebo drink, and drinks containing either caffeine $80 \mathrm{mg}$ or taurine $1000 \mathrm{mg}$, or both, on the performance of cognitive tests in 14 healthy volunteers (aged 18 to 21 years). Testing involved two cognitive tests, the Digit Span test from the WAIS and a focussed attention test, and 
Red Bull and cognitive function

commenced 45 minutes after drink consumption. No significant effects were observed between the five drinks on either of the cognitive tests. Tubbs and Tubbs (2011) compared the effects of RB $250 \mathrm{ml}$ to a placebo drink on manual dexterity using a standardised 9 hole peg test. Eighteen volunteers (aged 9 to 62 years) performed the test before and 30 minutes after RB $250 \mathrm{ml}$, and five volunteers (aged 10 to 69 years) performed the test before and after $250 \mathrm{ml}$ of water. There were no statistically significant benefits identified with RB. Howard and Marczinski (2010) studied the effects of various amounts of RB on a cued go/no-go task, in 80 volunteers aged 18 to 40 years. A parallel group design was used for five dosing conditions: RB $1.8 \mathrm{ml} / \mathrm{kg}$, RB $3.6 \mathrm{ml} / \mathrm{kg}, \mathrm{RB} 5.4 \mathrm{ml} / \mathrm{kg}$, placebo and no drink. Task performance was assessed prior to and 30 minutes after drinking began. Compared to placebo, all 3 doses of RB produced significant improvements in reaction times on the task, the lowest dose producing the greatest improvement. The same pattern was seen for selfrated mental fatigue. A number of studies have evaluated the effects of RB on driving performance. Horne and Reyner (2001) compared RB $500 \mathrm{ml}$ to a version of the product without caffeine, taurine and glucuronolactone, on the simulated driving performance of 12 sleep deprived healthy volunteers under 30 years of age (mean 24 years). Driving performance was evaluated in the afternoon on each of the two study days, following restriction of sleep the night before to a maximum of 5 hours. A baseline 30 minute driving session was completed prior to a 30 minute rest-period in which the study drinks were consumed, followed immediately by continuous 2 hour driving session. Driving performance was significantly improved by RB for the first 90 minutes of the 2 hour session. A secondary reaction time test was also improved, but only for the first 30 minutes. Reyner and Horne (2002) used a similar design in 12 healthy volunteers under 30 years (mean age 24), but the RB and control drink sizes were reduced to $250 \mathrm{ml}$. Again driving performance was superior with RB over the first 90 minutes of the post-drink session. Further, sleepiness as 
Red Bull and cognitive function

assessed using the Karolinska Sleepiness Scale was significantly reduced by RB over the first 90 minutes of the session. Mets et al. (2011) studied the effects of RB $250 \mathrm{ml}$ on prolonged car simulator driving in 24 healthy volunteers aged 21 to 35 years. Compared to a RB placebo prepared by the manufacturers (without taurine, caffeine, glucuronolactone, inositol, and vitamin B complex), RB significantly reduced the impairment in the primary driving parameter over the 2 hours following consumption, and for the first hour also significantly improved one of the three secondary driving quality outcomes. RB also significantly reduced subjective sleepiness as assessed with the Karolinska Sleepiness Scale over the 2 hour period.

Overall, the pattern of cognitive benefits for RB has been most consistent for sustained driving and alertness, with three of the eight studies cited above showing improvements on tests of attention, and one to immediate word recall. The present study was therefore conducted to comprehensively evaluate the profile of cognitive effects of RB across major domains of function. Additionally, in order to evaluate the role played by glucose in such effects, the sugar free version of RB was also studied. The primary outcomes were domain scores from the CogTrackTM System, an online set of tests of major aspects of attention, information processing, working and episodic memory, based closely on tasks which have previously shown sensitivity to detecting cognitive enhancement for a variety of substances, including caffeine and glucose (e.g. Haskell et al., 2005; Scholey and Kennedy, 2004).

\section{METHODS}

\section{Design}


Red Bull and cognitive function

This was a randomised, double-blind, placebo controlled, 3-way cross-over trial in 24 healthy young volunteers. The study was conducted at the NU-Food Research Centre, School of Agriculture, Food and Rural Development, Newcastle University. Approval for the study was given by the Faculty of Science, Agriculture and Engineering Ethics Committee, Newcastle University (Approval reference number 15-OKE-111).

\section{Volunteers}

Healthy young volunteers were recruited using opportunity sampling from Newcastle University and the surrounding area. The recruited study population comprised 18 females (mean age 22.1 years, SD 4.2, range 19 to 32) and 6 males (mean age 22.8 years, SD 5.34, range 19 to 33). The population mean (SD) height was $168 \mathrm{~cm}(7.9)$, weight $64.2 \mathrm{Kg}$ (12.6) and Body Mass Index (BMI) 22.7 (3.6). All volunteers were self-described as healthy, non-smokers and were screened to ensure they were fit to participate and free of food allergies and major psychiatric disorders. The Ethics Committee approved payment for participation which was in the form of 'gift cards'.

\section{Study Drinks}

The three study drinks were Red Bull $(250 \mathrm{ml})$, Red Bull Sugarfree $(250 \mathrm{ml})$ and a placebo $(250 \mathrm{ml})$, the ingredients of each drink are detailed below. Red Bull is known for having a very distinctive taste and appearance, thus when creating the placebo, it was essential that the taste of the three drinks was not distinguishable. In order to ensure this a Red Bull flavouring product was sourced which replicates the taste of Red Bull@ (http://www.fabulousflavours.co.uk/). To test that the flavouring was effective, a member of the laboratory staff at the site prepared the three study drinks, and four members of staff did 
Red Bull and cognitive function

a blind taste test. All four staff members determined that the products could not be individually identified.

To ensure blinding, the study drinks were prepared each day out of sight of the study staff day by a colleague who did not interact with the participants and played no other role in the study. This colleague held the randomisation code which was not shared until the study testing had been completed and the dataset finalised. Once the drinks were prepared, the study staff collected them, each drink being identified only by a participant number. The appearance of all three study drinks was fully concealed by the drinking apparatus, which comprised an opaque black plastic beaker; the drink being consumed via a straw placed through a hole in the lid.

The constituents of the drinks were as follows:

Red Bull $250 \mathrm{ml}$ :

Ingredients: Caffeine 80 mg, Taurine 1000 mg, B-group vitamins (B3, B5, B6, B12), Glucose 27 g, Alpine Spring Water (energydrink-uk.redbubull.com/ingredients-list)

Sugarfree Red Bull $250 \mathrm{ml}$ :

Ingredients: Caffeine 80 mg, Taurine 1000 mg, B-group vitamins (B3, B5, B6, B12), Aspartame and Acesulfame K, Alpine Spring Water (energydrinkuk.redbu-bull.com/red-bull-sugarfree-ingredients-list)

Placebo $250 \mathrm{ml}$ : 
Red Bull and cognitive function

Ingredients: Carbonated Water, Lemon Juice from concentrate (1\%), Citric Acid; Flavourings, Sodium Citrate; Sucralose, Acesulfame K, Potassium

Sorbate, Red Bull Flavouring which contains Propylene Glycol E1520 (0.23 $\mathrm{ml})$.

\section{Cognitive Function Testing}

Cognition was assessed using the $\operatorname{Cog}^{T r a c k}{ }^{\mathrm{TM}}$ System, an online set of nine cognitive tests (www.wesnes.com). Prior to the start of each test, all of the required task resources are downloaded within the browser, which ensures that the rates of stimuli presentation and the assessments of the speed and accuracy of each response are managed locally. It is not until the end of each task that the data are returned to the server. Overall, this procedure prevents poor or fluctuating internet connections from influencing the conduct of each test.

The instructions for each task are presented on the computer screen, and remain there until the volunteer initiates the test by pressing the right arrow keyboard key. The stimuli for all tasks are also presented on the screen. The in-task responses are made using the computer keyboard. The nine tasks take around 18 minutes to perform. The tasks are described below in the order in which they were administered:

- Immediate Word Recall: A list of 15 words is presented on the screen, at the rate of 1 word every 2 seconds, for the volunteer to remember. The volunteer is then given one minute to type all words which can be recalled, in any order, using the computer keyboard. The number of words correctly recalled and the number of words recalled which were not in the list (errors) are recorded.

- Pattern Separation: In this first stage, a series of 20 pictures of everyday scenes and objects is presented on the screen, at the rate of 1 picture every 3 seconds, for the 
Red Bull and cognitive function

volunteer to remember. The volunteer is instructed that the pictures will all be reshown later mixed with very similar ones.

- Simple Reaction Time: The volunteer is instructed to press the right arrow key on the keyboard as quickly as possible every time a stimulus is presented in the centre of the screen. The stimulus is a $4.5 \mathrm{~cm}$ by $3.5 \mathrm{~cm}$ image of a right facing arrow containing the word 'YES'. The volunteer is informed that only this stimulus will be presented and that it will remain there until a response is made. Throughout the test the volunteer is required to keep the right index finger resting lightly on the right arrow key. Fifty stimuli are presented with an inter-stimulus interval which varies randomly between 1 and 3.5 seconds. The speed of each response is recorded.

- Digit Vigilance: A target digit from 1 to 9 is randomly selected and constantly displayed to the right hand side of the screen. Digits are then presented one at a time in the centre of the screen at the rate of 150 per minute. The digits and the target digit are 2 $\mathrm{cm}$ wide by $3 \mathrm{~cm}$ high. The volunteer is required to press the right arrow key as quickly as possible every time a digit matches the target digit. Again the volunteer is required to keep the right index finger resting on the right arrow keyboard key throughout the task. A total of 450 digits is presented, with 15 target digits in each block of 150 digits. Correct detections, the speed of the detections and responses made in error (False Alarms) are recorded.

- Choice Reaction Time: The two possible stimuli in this task are either the right facing arrow used in Simple Reaction Time, or an equivalently sized left facing version of the arrow, with the word 'NO' in the middle. On each of 50 successive trials, one of these two stimuli is selected randomly (but with equal probability) and presented in the centre of the screen, remaining there until a response is made. The interval between successive trials varies randomly between 1 and 3.5 seconds. The volunteer is required 
Red Bull and cognitive function

to maintain the left and right index fingers resting lightly on the appropriate keyboard keys and to respond as quickly and accurately as possible. The accuracy and speed of each response is recorded.

- Spatial Working Memory: A 3 by 3 array of light bulbs is presented on the screen for a 10 second period. Four of the nine light bulbs are lit. The volunteer has to memorise the position of the lit bulbs. There are then 36 subsequent presentations of the 3 by 3 array, each time with only a single bulb lit. For each presentation the volunteer is required to decide whether or not the lit bulb was one of those lit in the original presentation, pressing the right keyboard arrow if it was, and the left if it was not. The images remain on the screen until a response is made, the volunteer again being required to maintain the index fingers resting on the left and right keyboard arrows, and to respond as quickly and accurately as possible. Over the 36 presentations, each of the nine bulbs is lit on four of them, the order being randomised, following presentation rules that the two same 'lit positions' should not be presented consecutively; and no more than 4 target or distractor stimuli should be presented consecutively. The accuracy and speed of each response is recorded.

- Numeric Working Memory: A series of 5 different digits is presented on the screen at the rate of 1 every 1.2 seconds. The volunteer is instructed to hold the digits in memory. This is followed by a series of 30 probe digits, each of which remains on the screen until a response is made. For each stimulus the volunteer has to indicate whether or not it was in the original series, pressing the right keyboard arrow if it was, and the left if it was not, as quickly and accurately as possible. The volunteer again maintains the index fingers resting on the left and right keyboard arrows throughout the task. In the series of 30 probe digits, each of the digits 0 to 9 is presented three times, in a randomised order, following the presentation rules that the same digit should not be presented 
Red Bull and cognitive function

consecutively and no more than 4 target or 4 distractor stimuli should be presented consecutively. The accuracy and speed of each response is recorded.

- Delayed Word Recall: The volunteer is given one minute to type as many of the words as can be recalled from the list presented earlier, in any order, using the computer keyboard. The number of words correctly recalled and the number of words recalled which were not in the original list (errors) are recorded.

- Word Recognition: The 15 original words plus 15 distractor words are presented one at a time in a randomised order. For each word the volunteer is required to indicate whether or not it was from the original list of words by pressing the right keyboard arrow if it was, and the left if it was not, as quickly as possible. Each word remains on the screen until a response is made. The volunteer again maintains the index fingers resting on the left and right keyboard arrows. The accuracy and speed of each response is recorded.

- Pattern Separation: In this second stage the original pictures plus the 20 very similar distractor pictures are presented one at a time in a counterbalanced order. Half of the original pictures are presented prior to the very similar distractor versions, and half afterwards. For each picture the volunteer has to indicate whether or not it was the precise picture shown earlier, pressing the right keyboard arrow if it was, and the left if it was not, as quickly and accurately as possible. Each picture remains on the screen until a response is made. The volunteer again maintains the index fingers resting on the appropriate keyboard arrows throughout the task. The accuracy and speed of each response is recorded.

The outcome measures from the nine CogTrack $^{\mathrm{TM}}$ tasks are presented in Table 1. Comparable versions of these nine tests with the same outcome measures have been used 
Red Bull and cognitive function

in other test systems, primarily the CDR System (e.g. Wesnes 2003; Wesnes et al., 1987; 2000). The factor structure of the measures from the nine CDR System tasks has been evaluated with principal component analysis (Wesnes et al., 2000), and composite scores based on this structure have been used repeatedly as outcomes in a variety of studies (e.g. Jones et al., 2012; Kennedy et al., 2002; Scholey and Kennedy, 2004; Wesnes 2015; Wesnes et al., 2000; 2003). For the three attention tasks (digit vigilance, simple and choice reaction time), the accuracy scores load together on one factor, and the speed scores on another. The three speed scores are summed to form a score named the Attentional Intensity Index, and the accuracy scores are combined into a score named the Sustained Attention Index. The accuracy scores from the two working memory and four episodic memory tasks are combined to form the Memory Capacity Index. Finally, the speed scores from the numeric working memory, spatial working memory, word recognition and pattern separation tasks load together on a single factor and are combined to create the Speed of Retrieval Index. Each of these four factors combines the same task measures as used in the CDR System to form the factor scores named Power of Attention, Continuity of Attention, Quality of Memory and Speed of Memory.

\section{Self-Ratings of Mood and Alertness}

Caffeine Research Visual Analogue Scales (Haskell et al., 2005; Rogers et al., 2003). This questionnaire is designed to capture mood changes associated with caffeine and consists of seven descriptors, "relaxed", "alert", "jittery", "tired", "tense", "headache", and "overall mood", each followed by a $100 \mathrm{~mm}$ horizontal line scale labelled "not at all" (lefthand end) and "extremely" (right-hand end), except for the line for overall mood which was 
Red Bull and cognitive function

labelled "very bad" and "very good". Volunteers were instructed to "mark the lines according to how you feel right now". Ratings were scored from 0 to 100.

Bond-Lader Visual Analogue Scales of Mood and Alertness (Bond and Lader, 1974). This widely used set of sixteen $100 \mathrm{~mm}$ visual analogue scales (VAS) yields three extensively validated factor scores: Alertness, Calmness and Contentment.

Profile of Mood States (POMS; McNair et al., 1992). This 65-item self-report questionnaire has been used extensively and yields 6 validated factor scores named Tension, Depression, Anger, Vigour, Fatigue and Confusion, plus an overall score named Total Mood Disturbance.

\section{Vital Signs and Blood Glucose}

Heart rate and blood pressure were measured using an automated blood pressure monitor (Omron M10-IT, Omron, UK). Capillary blood glucose was measured using a portable blood glucose monitor system (ACCU-CHEK On call-EZ, Roche Ltd, Basel, Switzerland).

\section{Caffeine Consumption}

To assess the caffeine consumption of the volunteers during the day prior to each laboratory visit, the Caffeine Intake Tool (Bühler et al., 2013) was administered.

\section{Procedure}

Once recruited volunteers were screened and gave informed consent. They were required to attend the laboratory at 8 a.m. on four occasions each separated by seven days. Volunteers were instructed to avoid alcohol for the 24 hours prior to arrival, as well as to 
Red Bull and cognitive function

have fasted and not consumed caffeine since 8 p.m. the preceding evening. On arrival a blood glucose (BG) sample was taken the volunteers were given a standardized breakfast which comprised of $30 \mathrm{~g}$ of Kellogg's bran flakes and $80 \mathrm{ml}$ of semi skimmed milk (all of which had to be consumed). The volunteers also described their consumption of caffeine containing products during the previous day. The first of these study days was a familiarisation session during which the volunteers performed the $\mathrm{CogTrack}^{\mathrm{TM}}$ System on four successive occasions to ensure that any effects of practice, familiarity or test anxiety had been overcome prior to the first study day (Goldberg et al., 2015; Wesnes and Pincock, 2002). Blood glucose samples were taken after each performance of the CogTrack ${ }^{\mathrm{TM}}$ System, and volunteers also completed the Caffeine research VAS, Bond-Lader VAS and POMS on a single occasion. Measurements of height and weight were taken to calculate Body Mass Index (BMI). During three study days, following breakfast baseline assessments of heart rate, blood pressure, the CogTrack ${ }^{\mathrm{TM}}$ System and the three self-rating scales were undertaken. Volunteers were then given the allocated treatment drink all of which had to be consumed within five minutes. The same assessment procedures were repeated at 30,60 and 90 minutes post consumption of the drink; with the exception of the Bond-Lader VAS and POMS which were only repeated at 90 minutes post dose. Blood glucose was measured on arrival at the laboratory, again after the baseline assessment but prior to the study drink, then 20,50, 80 and 110 minutes after the drink was consumed. The order of administration of the three drinks over the three study days was counterbalanced using the six possible sequences from Williams Latin Squares.

\section{Statistical Analyses}

All analyses were conducted using the SAS statistical package Version 9.2. Repeated Measure Mixed Model (MMRM) Analyses of Variance (ANOVA) were conducted on the change from baseline data. Study Drink (3 levels), Time of Testing (3 levels), Sequence (6 
Red Bull and cognitive function

levels), and the interaction between Study Drink and Time of Testing, were fitted as fixed factors. Volunteers were fitted as a random factor. The Compound Symmetry covariance structure was used. Degrees of Freedom were adjusted using the Kenward-Roger approximation. For measures which had significant main effects of treatment and/or interactions, between-condition comparisons were performed with t-tests using the SAS LSMeans statement. Cohen's d effect sizes were calculated to identify the magnitude of any differences between the dosing condition identified by the ANOVAs. The effect sizes were calculated by dividing the LSMean differences by the square root of the residual variance from the MMRM ANOVAs. Cohen's classification of effect sizes was adopted, $d=0.2, d=0.5$ and $d=0.8$ being the thresholds for small, medium and large effects respectively (Cohen, 1992). Further, the baseline scores over the three study conditions for each measure were compared using simple ANOVA to ensure there were no systematic variations over the study conditions. For blood glucose, the same MMRM ANOVA methodology was undertaken, but using the actual scores obtained on the six time points of each study day. In order to determine any relationships between the changes in blood glucose and the cognitive and mood measures, Pearson's r correlation coefficients were calculated.

\section{RESULTS}

The study was conducted between November 2015 and January 2016, with all 24 volunteers completing the three study days. In order to determine whether the blinding of the drinks had been successful, at the end of each testing day the volunteers were asked to guess which study drink they had consumed. Of the 72 ratings, 20 were 'don't know', 23 identified the correct drink and 29 the incorrect drink. Four of the volunteers correctly identified all thee drink's. The blinding procedure had thus been successful.

\section{Caffeine Consumption}


Red Bull and cognitive function

The caffeine containing products the volunteers reported taking on the day before laboratory visits are in descending order of frequency, coffee, cola type fizzy drinks, tea and chocolate bars. Only two volunteers reported not taking a caffeine containing product, each on only one of the four visits. The mean (SD) number taken was 1.61 (SD 0.77), the range being 0 to 4 . Two volunteers reported taking 4, each on a single occasion. None of the volunteers on any study session reported taking either an energy drink or shot on the preceding day.

\section{CogTrack $^{\mathrm{TM}}$}

For one subject, on the baseline of the first study day, an equipment malfunction resulted in an improbably poor score on the Digit Vigilance task, with only 1 of the 45 targets being detected. Over the four sessions of the training day conducted a week before, this subject had detected either 43 or 44 of the targets. As this error occurred pre-dose on the first study dosing day, it was decided to substitute the data for the Digit Vigilance task for those from the first training session, which had taken place at the same time of day. No other data substitutions were made.

On the final study day an issue with internet connectivity resulted in one of the four daily test sessions being unfinished for five volunteers. The missing data which occurred as a consequence are listed below:

Subject 10, RB, 30 minutes' post drink, Tasks missing: Choice reaction time, Spatial working memory, Numeric working memory, Delayed word recall, Delayed word recognition, Pattern separation

Subject 11, RB, 30 minutes' post drink, Tasks missing: Spatial working memory, Numeric working memory, Delayed word recall, Word recognition, Pattern separation 
Red Bull and cognitive function

Subject 12, Placebo, Pre-Drink, Tasks missing: Delayed word recognition, Pattern separation

Subject 13, RB, 30 minutes' post drink, Tasks missing: Spatial working memory, Numeric working memory, Delayed word recall, Word recognition, Pattern separation

Subject 14, RBSF, 30 minutes' post drink, Tasks missing: Numeric working memory, Delayed word recall, Word recognition, Pattern separation

No other data was lost for the 24 volunteers, and these data were not replaced. Thus from a total of 3,456 tasks performed in the study, the data for 22 tasks were missing, plus the score described above which was replaced with a training value. Thus $99.3 \%$ of the study data was successfully gathered.

None of the between condition comparisons of the pre-dosing baseline scores for the four composite CogTrack ${ }^{\mathrm{TM}}$ measures approached significance (all $\mathrm{p}>0.8$ ), and thus the MMRM ANOVAs were conducted as planned.

For the Attentional Intensity index, there was not a significant main effect of drink, nor of repeated post-drink test sessions, nor an interaction between the two. The same pattern was seen for the Sustained Attention Index. The overall change from pre-dose values for these two measures are presented in Figure 1. It can be seen that for the Attentional Intensity Index, performance declined over the post-drink period, these declines being significant for all three drinks (placebo $t=2.38, p=0.0186$; RBSF $t=2.01, p=0.0467$; $B$ $t=2.07, p=0.0406$ ). These declines exceeded small Cohen's $d$ effect sizes (placebo $=0.28$; RBSF $=0.23 ; \mathrm{RB}=0.24)$. The Sustained Attention Index showed a different pattern, improvements over baseline levels being seen, this improvement being statistically significant for $\mathrm{RB}(t=2.55, \mathrm{p}=0.0129)$, but not for placebo $(t=1.07, p=0.2862)$ or $\mathrm{RBSF}$ $(t=0.87, p=0.3877)$. This improvement over baseline with RB had a Cohen's $d$ effect size of 
Red Bull and cognitive function

0.34, thus exceeding the threshold for a small effect, unlike the other two conditions (both $<0.15)$. The between condition comparisons of RB to the other two drinks were nonsignificant (RB to Placebo: $t=1.17, p=0.2451 ; R B$ to RBSF: $t=1.33, p=0.1852$ ).

For the Memory Capacity Index, there was a significant main effect of drink $(F[2,178]=4.93$, $p=0.0083$ ), but no effect of repeated post-drink test sessions nor an interaction between the two. There was also a significant main effect for the Speed of Retrieval Index $(F[2,179]=4.92, p=0.0083)$, but again no effect of repeated post-drink test sessions, nor an interaction between the two. The pattern for Memory Capacity Index in Figure 2 can be seen for notably greater declines to occur over the post-drink period for both placebo and RBSF than with RB. These declines in relation to pre-drink levels were significant both for placebo $(t=2.56, p=0.0161)$ and RBSF $(t=2.49, p=0.0192)$, but not for $R B(t=0.38$, $p=0.7072$ ). The Cohen's $d$ effect sizes of the declines in placebo and RBSF were 0.56 and 0.54 respectively, with both being medium clinical effect sizes. Between condition comparisons identified that $\mathrm{RB}$ was significantly superior both to placebo $(t=2.76, p=0.0063)$ and RBSF $(t=2.68, p=0.0081)$, with close to medium effect sizes $(0.47$ and 0.45 respectively). As can be seen in Figure 2, for the Retrieval Speed Index the pattern was for increases in speed following the three drinks. This increase was significant for $\mathrm{RB}(\mathrm{t}=4.02$, $p=0.0002)$, but not for either placebo $(t=0.35, p=0.728)$ or $R B S F(t=1.46, p=0.1482)$. The effect size of the improvement over baseline with RB exceeded a medium effect size $(d=0.57)$. Between condition comparisons revealed the differences to RB to be significant compared both with placebo $(t=3.05, p=0.0026$, Cohen's $d=0.52)$ and RBSF $(t=2.16$, $p=0.032$, Cohen's $d=0.37$ ). There was no difference on this measure between placebo and RBSF (t=0.92, $\mathrm{p}=0.3581)$.

In order to examine the consistency of the effects detected on the four composite factor scores in relation to the various task measures which contribute to them, Table 2 has been 
Red Bull and cognitive function

produced. It can be seen that the direction of overall benefits for RB over placebo were seen on seven of the eight measures which contribute to the Memory Capacity Index, and all four for the Speed of Retrieval Index. The same general pattern was seen for RB in relation to RBSF for the Memory Capacity Index, and for three of four measures on the Speed of Retrieval Index.

\section{Mood scales}

Three of the seven items from the Caffeine Research VAS showed significant main effects from the MMRM ANOVAs, but again, no significant changes over the post-drinking test sessions nor interactions. These three items were Alert $[F(2,184)=7.87 p=0.0005]$, Tired $[F(2,184)=3.4 p=0.0356]$ and Jittery $[F(2,184)=5.6 p=0.0044]$.

The main effects from three of the Caffeine Research Scales which showed significant main effects of study drink are presented in Figure 3. The Alert item increased significantly over baseline for all drinks (placebo $t=3.32, p=0.0016$, $d=0.49$; RBSF $t=3.37, p=0.0014, d=0.49$; RB $t=7.25, p<0.0001, d=1.06$ ). The increase was over twice as large for $R B$ as for the other two drinks, RB producing a significantly greater increase than either placebo $(t=3.46$, $p=0.0007, d=0.58)$ or RBSF $(t=3.41, p=0.0008, d=0.57)$. For the VAS item Tired, significant decreases over baseline were seen both for $R B(t=4.94, p<0.0001, d=0.63)$ and RBSF $(t=4.31, p=<0.0001, d=0.55)$, with a small non-significant decrease the placebo drink $(t=1.73, p=0.0873, d=0.22)$. Between condition comparisons identified $R B$ to show a significantly larger decrease than placebo $(t=2.46, p=0.015, d=0.41)$, and for RBSF to show a significantly larger decrease than placebo $(t=1.98, p=0.0493, d=0.33)$. For the VAS item Jittery, large and significant increases over baseline were seen both for RB $(t=3.69$, $p=0.0012, d=0.97)$ and RBSF $(t=3.55, p=0.0016, d=0.93)$, a trend for an increase being 
Red Bull and cognitive function

seen with the placebo drink $(t=1.78, p=0.0873, d=0.47)$. Between condition comparisons identified RB to show a larger increase then placebo $(t=3.0, p=0.0031, d=0.5)$, as did RBSF $(t=2.78, p=0.0060, d=0.47)$.

No significant effects were seen on either the Bond-Lader scale or POMS, which were administered prior to the study drink and again at the end of the 90 minute testing session.

\section{Blood Glucose}

Blood Glucose $(B G)$ did not show a significant main effect of drink $[F(2,389)=2.59 p=0.076]$, but did show a significant interaction decline over the post-drink assessments $[F(3,389)=124.86 p<0.0001]$ as well as an interaction between drink type and the assessment time $[F(10,389)=2.25 p=0.0148]$. The $B G$ levels are presented in Figure 4, showing the sharp increase following breakfast, followed by declines thereafter in all conditions. The basis for the interaction was clearly the smaller decline in BG twenty minutes after consuming RB compared to that following both RBSF ( $t=4.08, p=<0.0001$, $d=1.19)$ and placebo $(t=4.17, p<0.0001, d=1.22)$. The $B G$ levels on arrival overlapped over the three study conditions, implying consistent adherence to the fasting requirement. To further support this, the mean (SD) BG levels (mmol/L) on arrival on the training day and also the three subsequent study days were as follows: Training Day, 5.10 (0.40); Day 1 , 5.05 (0.30); Day 2, 5.06 (0.29); Day 3, 5.12 (0.33).

\section{Vital Signs}

Heart rate showed a trend for a main effect between the three drinks $[F(2,181)=2.39$ $p=0.0941]$, a significant main effect of visit $[F(2,180)=4.75 p=0.0097]$ but no interaction between the two. From Figure 5 it can be seen that volunteers' heart rate dropped in all conditions post-dosing, with all three declines being significant (placebo $t=4.02, p=0.0003$, 
Red Bull and cognitive function

$d=0.7$; RBSF $t=2.46, p=0.0189, d=0.43 ; R B t=4.39, p<0.0001, d=0.78)$. The smaller decline following RBSF was significant compared with RB ( $t=2.07, p=0.0401, d=0.35)$ but not placebo $(t=1.64, p=0.103, d=0.27)$.

Systolic blood pressure showed a trend for a main effect of study drink $[F(2,183)=2.99$ $p=0.0527]$, but no changes over time nor an interaction. The pattern was for a small and non-significant increase following placebo $(t=1.27, p=0.2125, d=0.22)$ and significant increases both for RBSF $(t=2.8, p=0.008, d=0.48)$ and $R B(t=3.6, p=0.0009, d=0.62)$. The increase with $R B$ was significantly greater than that with placebo $(t=2.41, p=0.0172, d=0.4)$, no other comparisons were significant.

Diastolic blood pressure showed a highly significant main effect of study drink $[F(2,181)=8.18 p=0.0004]$, but no changes over time nor an interaction. The pattern for this measure was for a small and non-significant increase following $R B(t=1.52, p=0.136$, $d=0.24)$ with significant increases both for RBSF $(t=5.5, p<0.0001, d=0.89)$ and placebo $(t=4.72, p<0.0001, d=0.75)$. Compared to $R B$, the greater increases in diastolic blood pressure were significant for both $\operatorname{RBSF}(t=3.83, p=0.0002, d=0.64)$ and placebo $t=3.04$, $p=0.0027, d=0.51)$

\section{Blood glucose, mood and cognitive function}

Over the post-dosing assessments following RB administration, there were no significant correlations between the changes in BG and any of the mood or performance measures. Following RBSF, only tiredness correlated with the drop in BG $(r=-0.32, p=0.0088)$. 
Red Bull and cognitive function

However, following the placebo drink the drops in BG correlated with the Caffeine VAS items Alert $(r=0.35, p=0.0029)$ and Tired $(r=-0.4, p=0.0004)$, and three of the four performance indices: Attentional Intensity Index $(r=-0.26, p=0.029)$, Sustained Attention Index $(r=0.28, p=0.0193)$, and Memory Capacity Index $(r=0.31, p=0.0086)$; but not the Retrieval Speed Index ( $r=-0.02, p=0.8701)$.

\section{DISCUSSION}

The major finding from this study was the that RB had positive effects upon memory performance in healthy young volunteers. These effects occurred during a 90 minute testing session which began 30 minutes following the drink. The Memory Capacity Index, a measure representing the combined accuracy scores from two working memory and four episodic memory tasks was significantly superior following RB compared both to a placebo drink and RBSF. Further, the Retrieval Speed Index, a measure of the speed of information retrieval from the two working memory tasks and the two episodic recognition tasks also showed significant beneficial effects of RB compared to RBSF and placebo. These comprehensive improvements, reflecting both the accuracy and speed of performance in the working and episodic memory tasks, were also of a magnitude which can be considered clinically relevant. The Cohen's d effect size of the improvement of RB over placebo was 0.47 for Memory Capacity Index and 0.52 for the Retrieval Speed Index, which puts them in the range of clinically meaningful differences for neuropsychological measures (Harvey 2012).

The absence of any noticeable difference in the Memory Capacity Index between RBSF and placebo does suggest that the positive effects of RB are related to the glucose content of the product. Such an effect is consistent with previous research on the effects of glucose 
Red Bull and cognitive function

on memory at the levels seen in RB. Smith et al ${ }_{2=}(2011)$ in their comprehensive review of the 'glucose memory facilitation effect' concluded that: "In summary, the ingestion of oral glucose is known to enhance cognitive performance under specific conditions. Glucose has been most reliably associated with the modulation of verbal episodic memory. In healthy young adults and adolescents, encoding of memory materials under conditions of increased cognitive demand appears to be critical." (page 781). It is reasonable to propose that the present study which involved the administration of six memory tests every 30 minutes over a 90 minute period fulfilled the criterion of increased mental demand. In considering the potential mechanisms underlying these effects, Smith et al. (2011) concluded: "The most robust of these theories in terms of empirical evidence is the hypothesis that glucose enhances memory via its effects on ACh synthesis" (page 781). Riby (2004) in a metaanalysis of 104 studies of the effects of glucose on cognitive function, identified that in younger adults, tests of verbal episodic, visuospatial episodic and working memory showed highly significant overall benefits of glucose, whereas no significant effects were seen for other task groups including attention, executive, motor, implicit memory and semantic retrieval. Further, the optimum dose for improving memory was $25 \mathrm{~g}$, with smaller benefits at the higher doses studied, most commonly $50 \mathrm{~g}$. Although significant correlation in the placebo condition between the decline in the Memory Capacity Index and the falling blood glucose levels does link blood glucose to this measure of memory, the absence of any such correlation in the RBSF condition argues against glucose as being the sole basis for this effect, and suggests that some form of interaction between glucose and the various other constituents of RB could be the basis for the beneficial effect of the product seen in the present study. Scholey and Kennedy (2004), in a similarly designed study in young volunteers, used the CDR System to compare the effects of placebo, glucose $37.5 \mathrm{~g}$, caffeine $75 \mathrm{mg}$, and the combination of these doses of glucose and caffeine. On the Quality 
Red Bull and cognitive function

of Memory score, upon which the Memory Capacity Index is based, it was identified that the combination significantly outperformed the other conditions, with no benefits of either caffeine or glucose alone being detected. Adan and Serra- Grabulosa (2010) conducted a similarly designed study with glucose $75 \mathrm{~g}$ and caffeine $80 \mathrm{mg}$, and found the combination to significantly outperform both the individual doses as well as placebo on the Rey Auditory Verbal Learning task, and as with Scholey and Kennedy (2004), found no benefits with the individual doses of glucose and caffeine. These findings further caution against attributing the benefits of RB on memory in the present study solely to the glucose content of the product. Also it must be borne in mind that in the RB condition, plasma glucose was only $\underline{\text { higher at the start of the first of the three testing sessions. }}$

In terms of the self-ratings of mood states, RB produced significant increases in 'alertness' compared with placebo and RBSF, in both cases these were of medium to large effect sizes. In a previous study which used the same questionnaire to study the effects of caffeine alone (75 $\mathrm{mg}$ and $150 \mathrm{mg}$ ) in regular caffeine consumers, no significant increase in 'alertness' was identified (Haskell et al., 2005). This suggests that caffeine alone may not be sufficient to increase alertness. However, Haskell et al., (2005) found the VAS item 'tired' to be significantly decreased by both doses of caffeine, which is consistent with the reductions in this item seen with RB and RBSF compared to placebo in the present study, and points to this reduction being related to the caffeine content of the drinks. The same appears to be the case for the VAS item 'jittery', both RB and RBSF increased the rating of this item over placebo, which again is consistent with previous work with caffeine (Haskell et al., 2005; Rogers et al., 2003). This pattern suggests that glucose, possibly together with caffeine, improves alertness with RB, whereas caffeine primarily reduces 'tiredness'. 
Red Bull and cognitive function

As described in the Introduction, some but by no means all of the previous studies with RB have found aspects of attention to be improved by the product. In the present study there was some suggestions of a positive effect of RB on sustained attention, but this was not a robust effect and the overall effects of RB on attention remain to be firmly demonstrated. The tests employed in this study when used in comparably designed and powered studies have shown them to be sensitive to the positive attentional effects of a variety of substances including caffeine $75 \mathrm{mg}$ (Haskell et al., 2005), glucose $25 \mathrm{~g}$ (Jones et al., 2012) and a combination of glucose $37.5 \mathrm{~g}$ and caffeine $75 \mathrm{mg}$ (Scholey and Kennedy, 2004). This suggests that test sensitivity and sample size is not the reason for the absence of effects of RB on attention in the present study. Giles et al. (2012) identified that taurine 2000 mg impaired speed on a Choice Reaction Time task. Peacock et al. (2013) found a trend towards impaired performance in a stimulus degradation task when taurine $1000 \mathrm{mg}$ was co administered with caffeine $80 \mathrm{mg}$, compared with caffeine alone. This suggests that taurine may to some extent counteract the effects of caffeine, which could be related to the absence of attentional benefits of RB in the present study.

Weaknesses of the study include not obtaining information from the volunteers about their general energy drink and caffeine consumption (beyond the previous day of each study visit), as well as not taking salivary caffeine samples on arrival at the laboratory to check compliance with caffeine abstinence. From the caffeine intake reports the day prior to each session, it was evident that all subjects were caffeine consumers. Further, as the highest consumption reported was consuming four caffeine containing items, which only occurred on two of 96 possible occasions, this implies that the volunteers were not heavy caffeine users. Finally, as none reported taking either an energy drink or shot on any of the days previous to the testing days, it would appear that the volunteers were not regular users of such products. 
Red Bull and cognitive function

\section{CONCLUSIONS}

In conclusion this study has shown that RB produces marked and highly significant improvements to the accuracy and speed of working and episodic memory which were not seen with RBSF or placebo. RB also improved self-ratings of alertness, while both RB and RBSF reduced tiredness. There were no clear effects of RB or RBSF in the measures of attention. Although the beneficial effects of RB on memory are consistent with previous effects seen with the same general amount of glucose in healthy young volunteers, it should be noted that the plasma glucose levels were only higher during the first of the three post-drink test sessions. Further, it must be borne in mind that the memory benefits could result from an interaction between glucose and the other constituents of RB, as opposed to an effect of glucose alone. These beneficial effects of RB on memory are consistent with previous effects seen with the same general amount of glucose in healthy young volunteers. However it must be borne in mind that the memory benefits could result from an interaction between glucose and the other constituents of RB, as opposed to an effect of glucose alone. 
Red Bull and cognitive function

\section{REFERENCES}

Alford C, Cox H, Wescott R et al. (2001) The effects of Red Bull Energy Drink on human performance and mood. Amino Acids 21: 139-150.

Adan, A. and Serra-Grabulosa, J. M. (2010) Effects of caffeine and glucose, alone and combined, on cognitive performance. Human Psychopharmacology: Clinical and Experimental, 25(4), 310-317.

Bond A and Lader M (1974) The use of analogue scales in rating self-rated feelings. British Journal of Medical Psychology; 47: 211-218.

Cohen J (1992) A Power Primer. Psychological Bulletin 112: 155-159.

Bühler E, Lachenmeier DW, Schlegel K and Winkler G (2013) Development of a tool to assess the caffeine intake among teenagers and young adults. Ernahrungs Umschau 61: 58-63

Gendle MH, Smucker DM, Stafstrom JA et al. (2009) Attention and Reaction Time in University Students Following the Consumption of Red Bull囚. The Open Nutrition Journal 3: 8-10

Giles GE, Mahoney CR, Brunyé TT et al. (2012) Differential cognitive effects of energy drink ingredients: Caffeine, taurine, and glucose. Pharmacology, Biochemistry and Behavior 102: 569-577.

Goldberg TE, Harvey PD, Wesnes KA et al. (2015). Practice Effects Due to Serial Cognitive Assessment: Implications for Preclinical Alzheimer's Disease 
Red Bull and cognitive function

Randomized Controlled Trials. Alzheimer's \& Dementia: Diagnosis, Assessment \& Disease Monitoring 1: 103-111.

Harvey P (2012) Clinical applications of neuropsychological assessment. Dialogues in Clinical Neuroscience 14: 91-99.

Haskell CF, Kennedy DO, Wesnes KA et al. (2005) Cognitive and mood improvements of caffeine in habitual consumers and habitual non-consumers of caffeine. Psychopharmacology 179: 813-825.

Horne JA and Reyner LA (2001) Beneficial effects of an "energy drink" given to sleepy drivers. Amino Acids 20: 83-89.

Howard MA and Marczinski CA (2010) Acute effects of a glucose energy drink on behavioral control. Experimental and Clinical Psychopharmacology.18, 6: 553561.

Ishak WW, Ugochukwu C, Bagot K, et al. (2012) Energy drinks: Psychological Effects and Impact on Well-being and Quality of Life - A Literature Review. Innov Clin Neurosci. 9: 25-34.

Jones EK, Sünram-Lea SI and Wesnes KA. (2012) Acute ingestion of different macronutrients differentially enhances aspects of memory and attention in healthy young adults. Biological Psychology 89: 477-468.

Kammerer M, Jaramillo JA, García A et al. (2014) Effects of energy drink major bioactive compounds on the performance of young adults in fitness and cognitive tests: a randomized controlled trial. Journal of the International Society of Sports Nutrition 11: 44. 
Red Bull and cognitive function

Kennedy DO, Scholey AB, Tildesley NTJ, (2002) Modulation of mood and cognitive performance following acute administration of Melissa officinalis (lemon balm) Pharmacology, Biochemistry \& Behavior 72: 953-964.

McNair DM, Lorr M and Droppleman LF (1992) EdITS Manual for the Profile of Mood States. San Diego, CA, EdITS.

Mets MAJ, Ketzer S, Blom C et al. (2011) Positive effects of Red Bull囚 Energy Drink on driving performance during prolonged driving. Psychopharmacology 214: $737-745$.

Peacock A, Martin FH and Carr A (2013) Energy drink ingredients. Contribution of caffeine and taurine to performance outcomes. Appetite 64: 1-4.

Reyner LA and Horne JA (2002) Efficacy of a 'functional energy drink' in counteracting driver sleepiness. Physiol Behav 75: 331-335.

Riby LM (2004) The impact of age and task domain on cognitive performance: a meta-analytic review of the glucose facilitation effect. Brain Impairment 5: 145-165.

Rogers PJ, Martin J, Smith C, Heatherley SV et al. (2003) Absence of reinforcing, mood and psychomotor performance effects of caffeine in habitual nonconsumers of caffeine. Psychopharmacology 167: 54-62.

Scholey AB and Kennedy DO (2004) Cognitive and physiological effects of an "energy drink": an evaluation of the whole drink and of glucose, caffeine and herbal flavouring fractions. Psychopharmacology. 176: 320-330.

Smith MA, Riby LM, Eekelen JA et al. (2011) Glucose enhancement of human memory: a comprehensive research review of the glucose memory facilitation 
Red Bull and cognitive function

effect. Neurosci Biobehav Rev 35: 770-783.

Tubbs I and Tubbs RS (2011) Does Drinking Red Bull ${ }^{\mathrm{TM}}$ Increase Manual Dexterity? Biomedicine International 2: 2-4

Wesnes KA (2003) The Cognitive Drug Research computerised assessment system: Application to clinical trials. In: De Deyn P, Thiery E, D’Hooge R (Eds.) Memory: Basic Concepts, Disorders and Treatment. Uitgeverij Acco: Leuven, pp. $453-472$.

Wesnes KA (2015) The effects on cognitive function in healthy volunteers of 28 days treatment with a combination of Acetyl-L-Carnitine, Vinpocetine and Huperzine A. J Nutr Health Aging 19 (Supplement 1): 24-25.

Wesnes KA, Luthringer R, Ambrosetti $L$ et al. (2003) The effects of a combination of Panax ginseng, vitamins and minerals on mental performance, mood and physical fatigue in nurses working night shifts: a double-blind, placebo controlled trial. Current Topics in Nutraceutical Research 1: 169-174.

Wesnes KA and Pincock C (2002) Practice effects on cognitive tasks: a major problem? Lancet Neurology 1: 473.

Wesnes KA, Simpson PM and Kidd AG (1987) The use of a scopolamine model to study the nootropic effects of tenilsetam (CAS 997) in man. Medical Science Research 15: 10631064.

Wesnes KA, Ward T, McGinty A et al. (2000) The memory enhancing effects of a Ginkgo biloba/Panax ginseng combination in healthy middle aged volunteers. Psychopharmacology 152: 353-361. 
Red Bull and cognitive function

Table 1: Core measures from the $\operatorname{Cog}$ rack $^{T \mathrm{M}}$ tasks

\begin{tabular}{|l|l|}
\hline Task & Measure \\
\hline Simple Reaction Time & Speed (msec) \\
\hline Digit Vigilance & Targets detected (\%) \\
& False Alarms (\#) \\
& Speed of target detection (msec) \\
\hline Choice Reaction Time & $\begin{array}{l}\text { Accuracy (\%) } \\
\text { Speed of correct responses (msec) }\end{array}$ \\
\hline Numeric Working Memory & $\begin{array}{l}\text { Original Stimuli - Accuracy (\%) } \\
\text { New Stimuli - Accuracy (\%) } \\
\text { Speed of correct responses (msec) }\end{array}$ \\
\hline Spatial Working Memory & $\begin{array}{l}\text { Original Stimuli - Accuracy (\%) } \\
\text { New Stimuli Accuracy (\%) } \\
\text { Speed of correct responses (msec) }\end{array}$ \\
\hline Immediate Word Recall & Words correctly recalled - Accuracy (\%) \\
& Errors (\#) \\
\hline Delayed Word Recall & Words correctly recalled - Accuracy (\%) \\
& Errors (\#) \\
\hline Word Recognition & Original Stimuli - Accuracy (\%) \\
& Lure Stimuli Accuracy (\%) \\
& Speed of correct responses (msec) \\
\hline Pattern Separation & Original Stimuli - Accuracy (\%) \\
& New Stimuli Accuracy (\%) \\
& Speed of correct responses (msec) \\
\hline
\end{tabular}


Red Bull and cognitive function

Table 2: The overall change from baseline scores for each of the individual items which contribute to the four composite factor scores which were the major study outcomes

\begin{tabular}{|c|c|c|c|c|c|}
\hline \multirow[t]{2}{*}{ Factor Score } & \multirow[t]{2}{*}{ Task } & \multirow[t]{2}{*}{ Measure (units) } & Placebo & Red Bull Sugarfree & Red Bull \\
\hline & & & $\begin{array}{c}\text { Mean } \\
\text { (Standard Error) }\end{array}$ & $\begin{array}{c}\text { Mean } \\
\text { (Standard Error) }\end{array}$ & $\begin{array}{c}\text { Mean } \\
\text { (Standard Error) }\end{array}$ \\
\hline \multirow{3}{*}{$\begin{array}{l}\text { Attentional } \\
\text { Intensity Index }\end{array}$} & Simple Reaction Time & Speed $(\mathrm{msec}) *$ & $14.1(8.3)$ & $22.8(8.3)$ & $18.4(8.3)$ \\
\hline & Digit Vigilance & Speed of target detection (msec) ${ }^{*}$ & $13.7(4.6)$ & $5.8(4.6)$ & $4.7(4.6)$ \\
\hline & Choice Reaction Time & Speed of correct responses (msec) ${ }^{*}$ & $0.6(6.0)$ & $-4.6(6.0)$ & $1.1(6.0)$ \\
\hline \multirow{3}{*}{$\begin{array}{l}\text { Sustained } \\
\text { Attention Index }\end{array}$} & \multirow[t]{2}{*}{ Digit Vigilance } & Targets detected $(\%)$ & $1.14(1.3)$ & $-0.12(1.3)$ & $1.70(1.3)$ \\
\hline & & False Alarms (\#) * & $-0.18(0.3)$ & $-0.10(0.3)$ & $-0.22(0.3)$ \\
\hline & Choice Reaction Time & Accuracy $(\%)$ & $-0.03(0.5)$ & $0.56(0.5)$ & $0.60(0.5)$ \\
\hline \multirow{8}{*}{$\begin{array}{l}\text { Memory Capacity } \\
\text { Index }\end{array}$} & Numeric Working Memory & Overall Accuracy (\%) & $-2.59(1.8)$ & $2.46(1.8)$ & $-0.24(1.9)$ \\
\hline & Spatial Working Memory & Overall Accuracy (\%) & $-3.61(2.6)$ & $-0.90(2.6)$ & $-0.62(2.7)$ \\
\hline & \multirow[t]{2}{*}{ Immediate Word Recall } & Words correctly recalled - Accuracy (\%) & $-1.85(3.0)$ & $-3.06(3.0)$ & $-0.09(3.0)$ \\
\hline & & Errors (\#) * & $-0.06(0.1)$ & $-0.11(0.1)$ & $0.19(0.1)$ \\
\hline & \multirow[t]{2}{*}{ Delayed Word Recall } & Words correctly recalled - Accuracy (\%) & $-12.5(3.2)$ & $-9.1(3.2)$ & $-7.6(3.3)$ \\
\hline & & Errors (\#) * & $0.42(0.2)$ & $0.28(0.2)$ & $0.38(0.2)$ \\
\hline & Word Recognition & Overall Accuracy (\%) & $-12.5(3.2)$ & $-9.06(3.2)$ & $-7.58(3.3)$ \\
\hline & Pattern Separation & Overall Accuracy (\%) & $-0.38(3.1)$ & $-15.5(3.0)$ & $5.95(3.1)$ \\
\hline \multirow{4}{*}{$\begin{array}{l}\text { Speed of Retrieval } \\
\text { Index }\end{array}$} & Numeric Working Memory & Speed of correct responses (msec) ${ }^{*}$ & $-13.9(12.5)$ & $-16.5(12.6)$ & $-37.3(12.7)$ \\
\hline & Spatial Working Memory & Speed of correct responses (msec) ${ }^{*}$ & $-30.6(13.7)$ & $-42.7(13.7)$ & $-33.2(13.9)$ \\
\hline & Word Recognition & Speed of correct responses (msec) ${ }^{*}$ & $22.9(14.4)$ & $7.7(14.2)$ & $-14.0(14.3)$ \\
\hline & Pattern Separation & Speed of correct responses (msec) * & $8.2(12.8)$ & $2.0(12.6)$ & $-48.6(12.7)$ \\
\hline
\end{tabular}

Note $*$ indicates positive change in score reflects impaired performance 


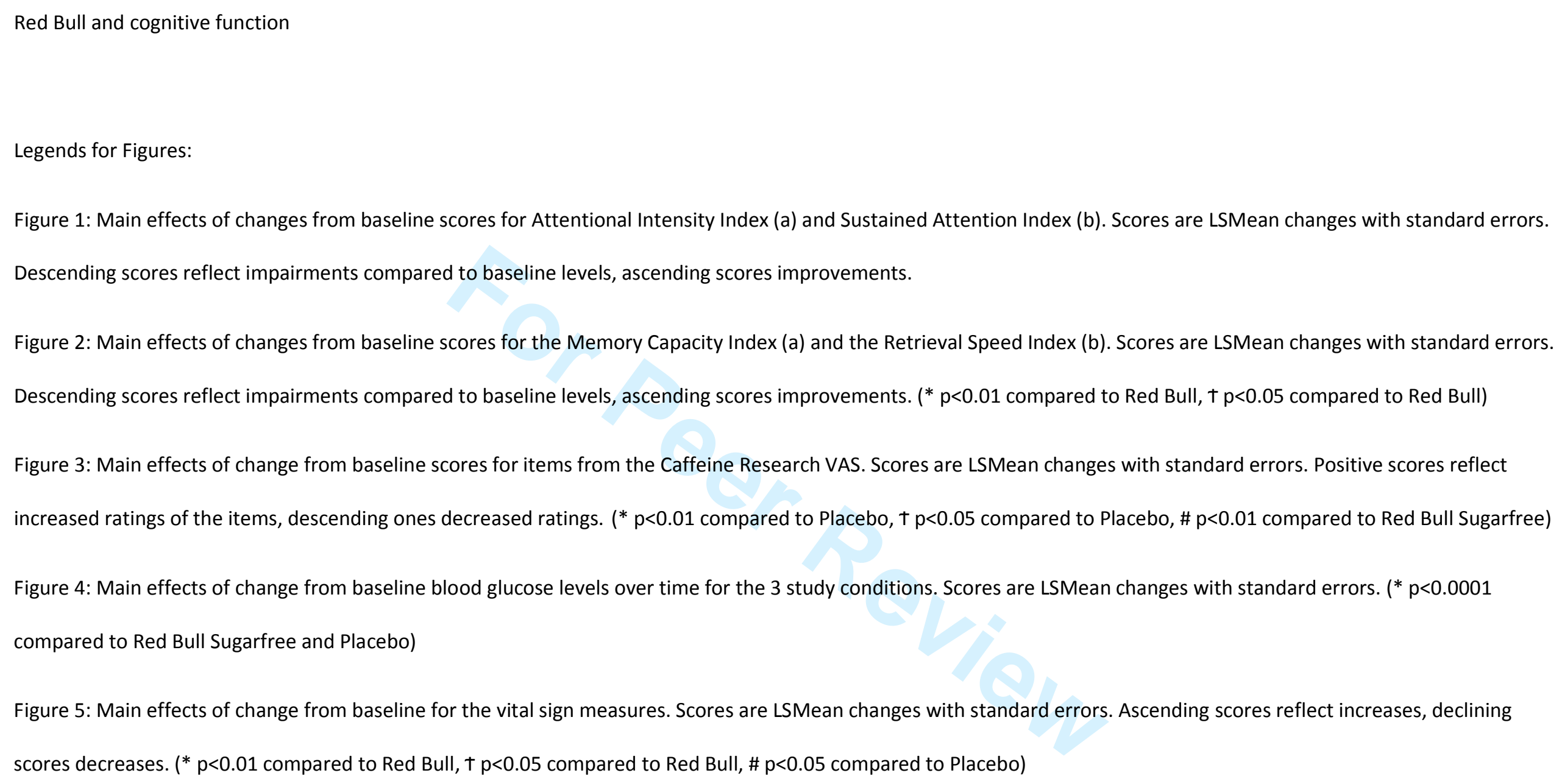




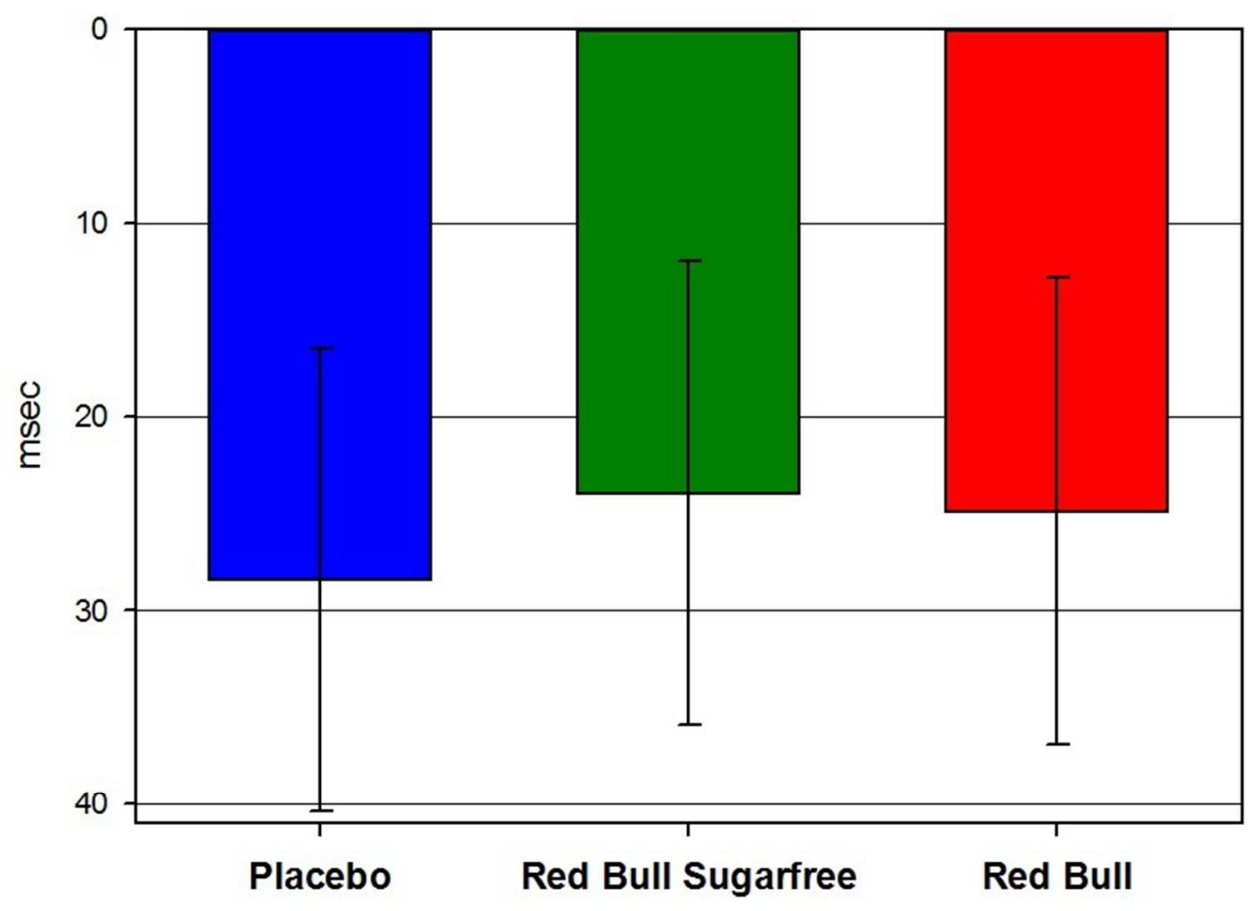

$147 \times 109 m m(150 \times 150$ DPI $)$

35

36

37

38

39

40

41

42

43

44

45

46

47

48

49

50

51

52

53

54

55

56

57

58

59

60

http://mc.manuscriptcentral.com/jop 
1

2

3

4

5

6

7

8

9

10

11

12

13

14

15

16

17

18

19

20

21

22

23

24

25

26

27

28

29

30

31

32

33

34

35

36

37

38

39

40

41

42

43

44

45

46

47

48

49

50

51

52

53

54

55

56

57

58

59

60

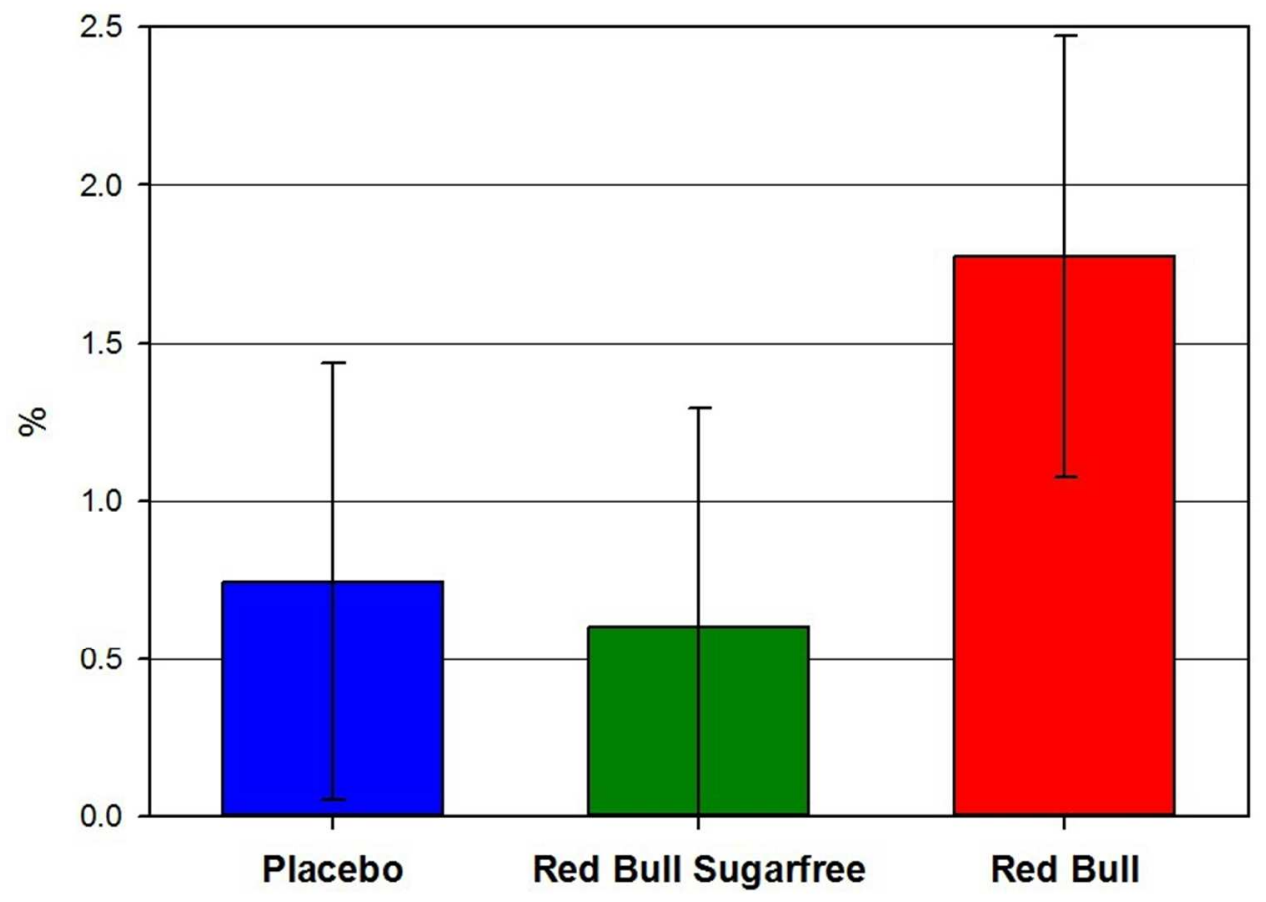

$148 \times 109 m m(150 \times 150$ DPI $)$

http://mc.manuscriptcentral.com/jop 


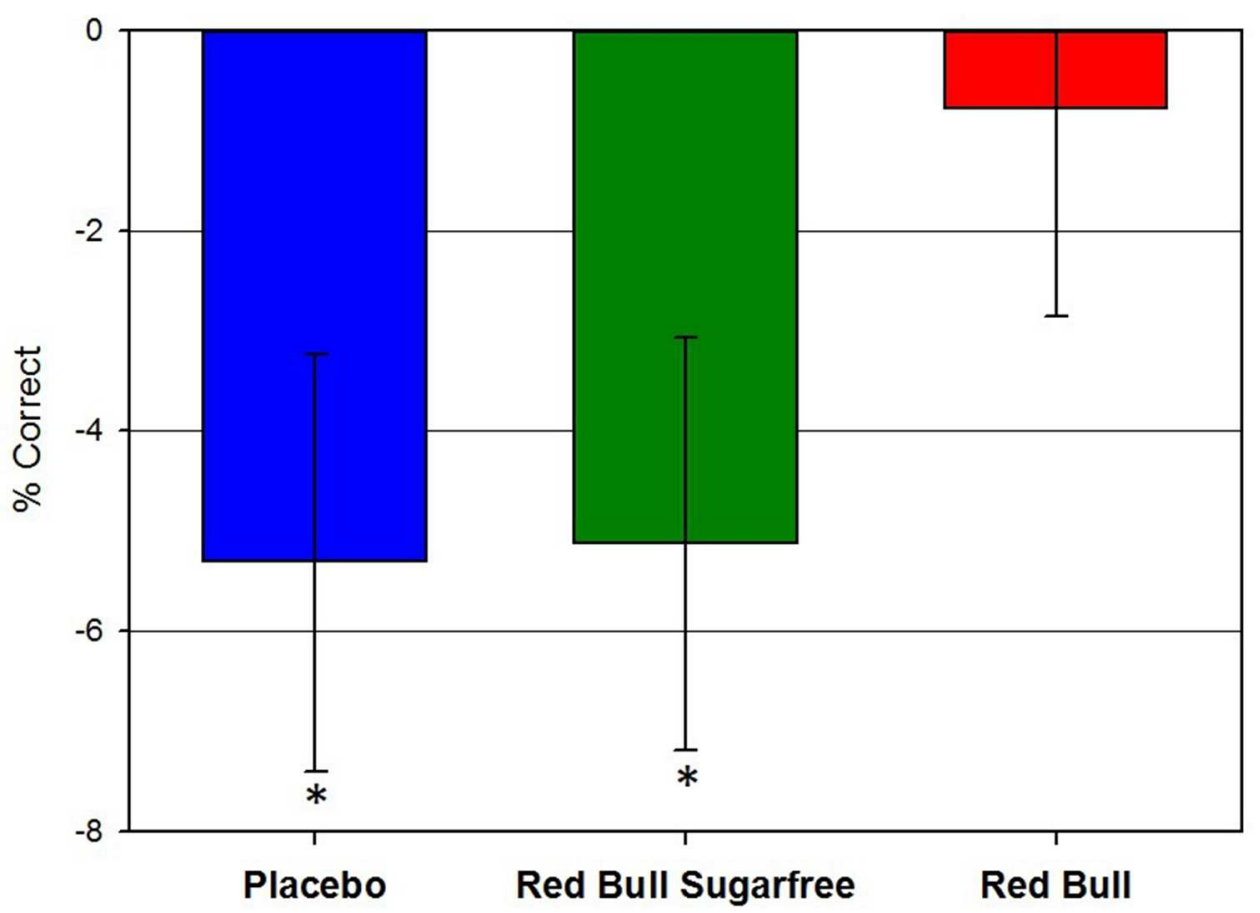

$146 \times 109 \mathrm{~mm}(150 \times 150 \mathrm{DPI})$

http://mc.manuscriptcentral.com/jop 


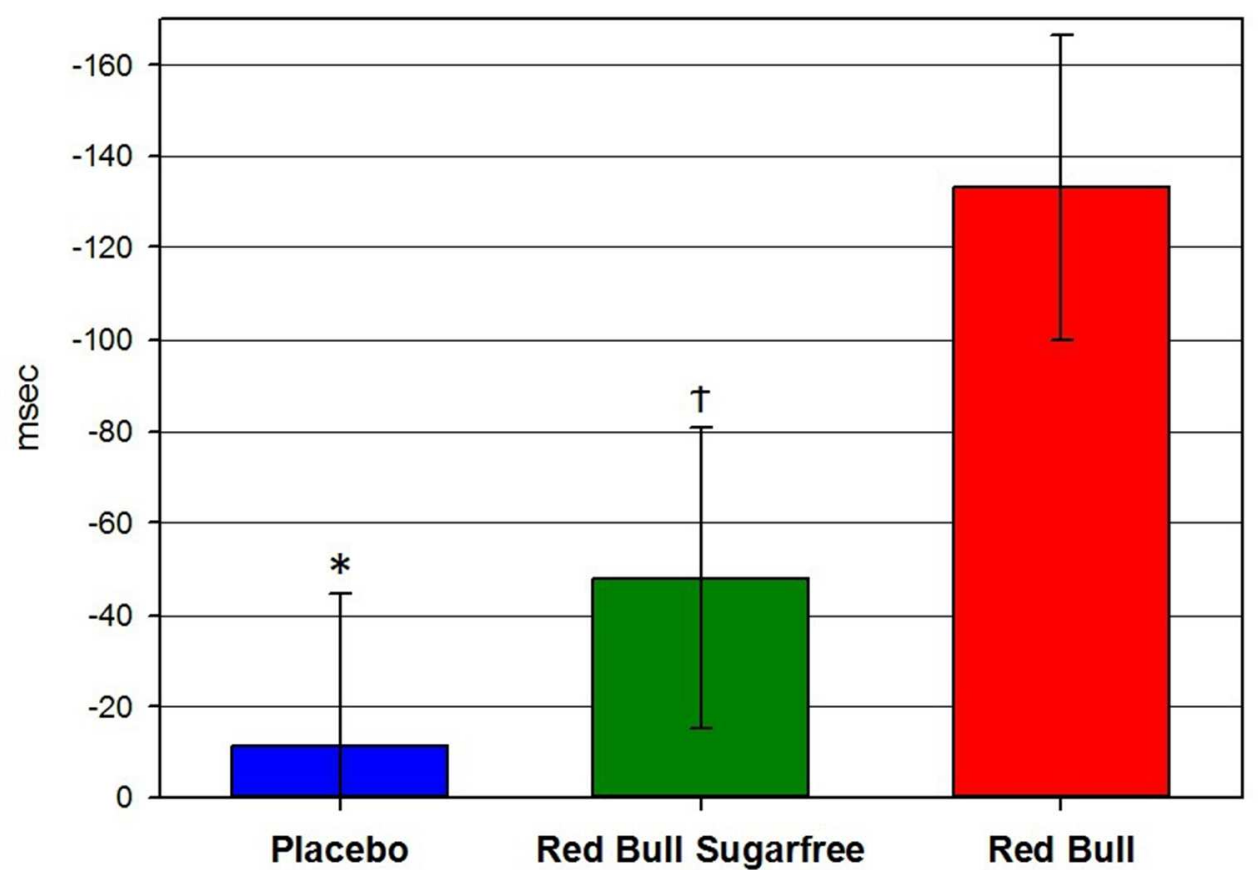

$150 \times 109 \mathrm{~mm}(150 \times 150 \mathrm{DPI})$ 


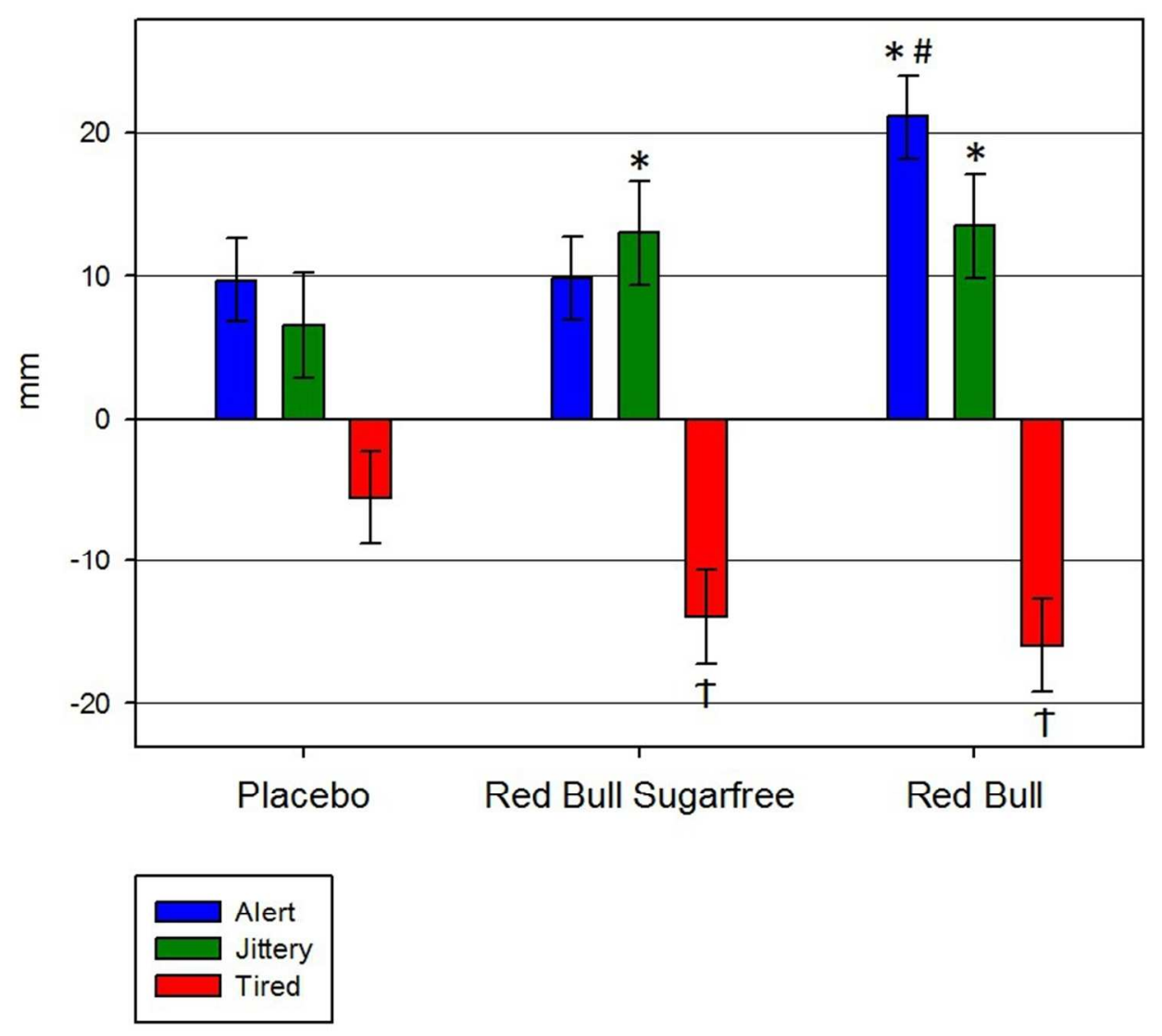

$149 \times 132 \mathrm{~mm}(150 \times 150 \mathrm{DPI})$

http://mc.manuscriptcentral.com/jop 


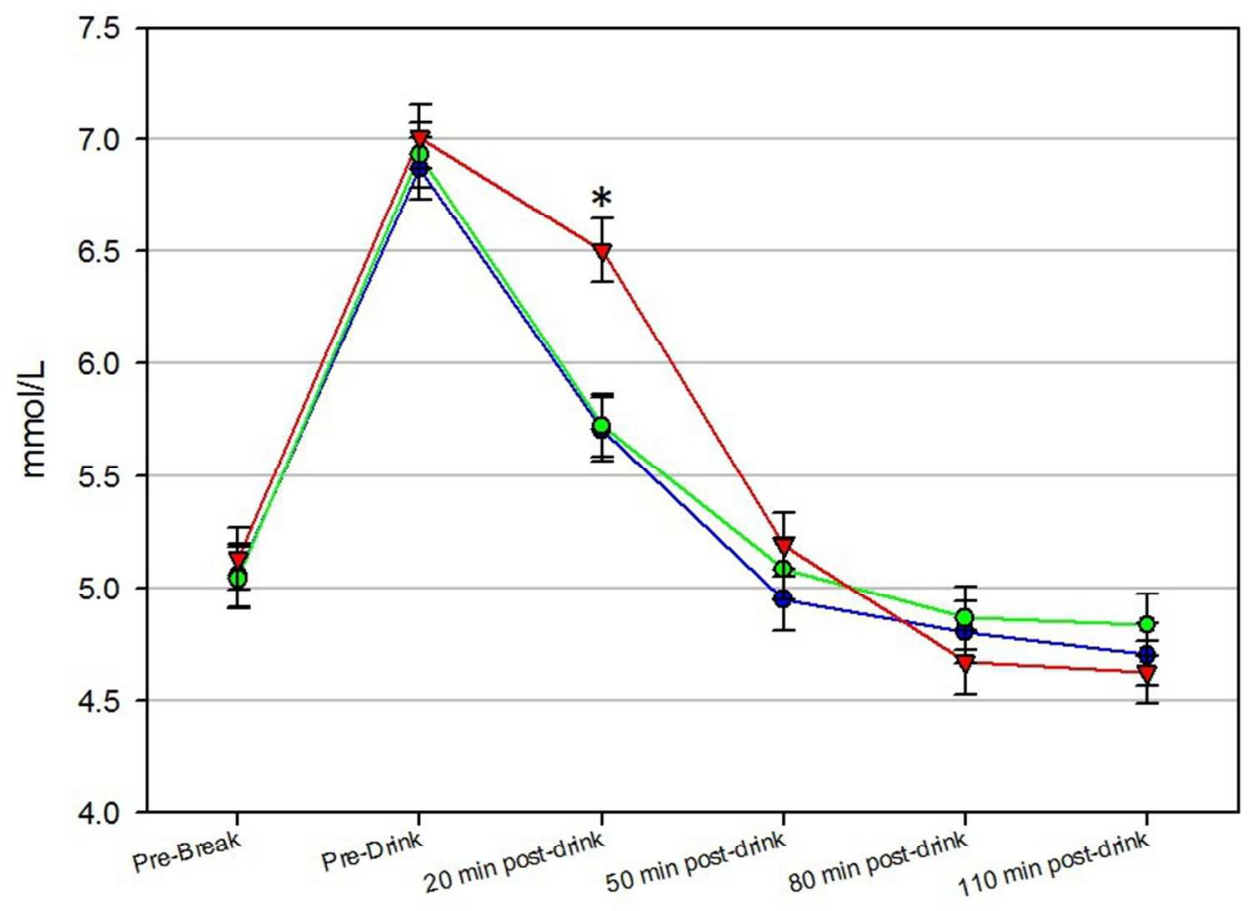

Time of Sampling

$149 \times 129 \mathrm{~mm}(150 \times 150 \mathrm{DPI})$ 


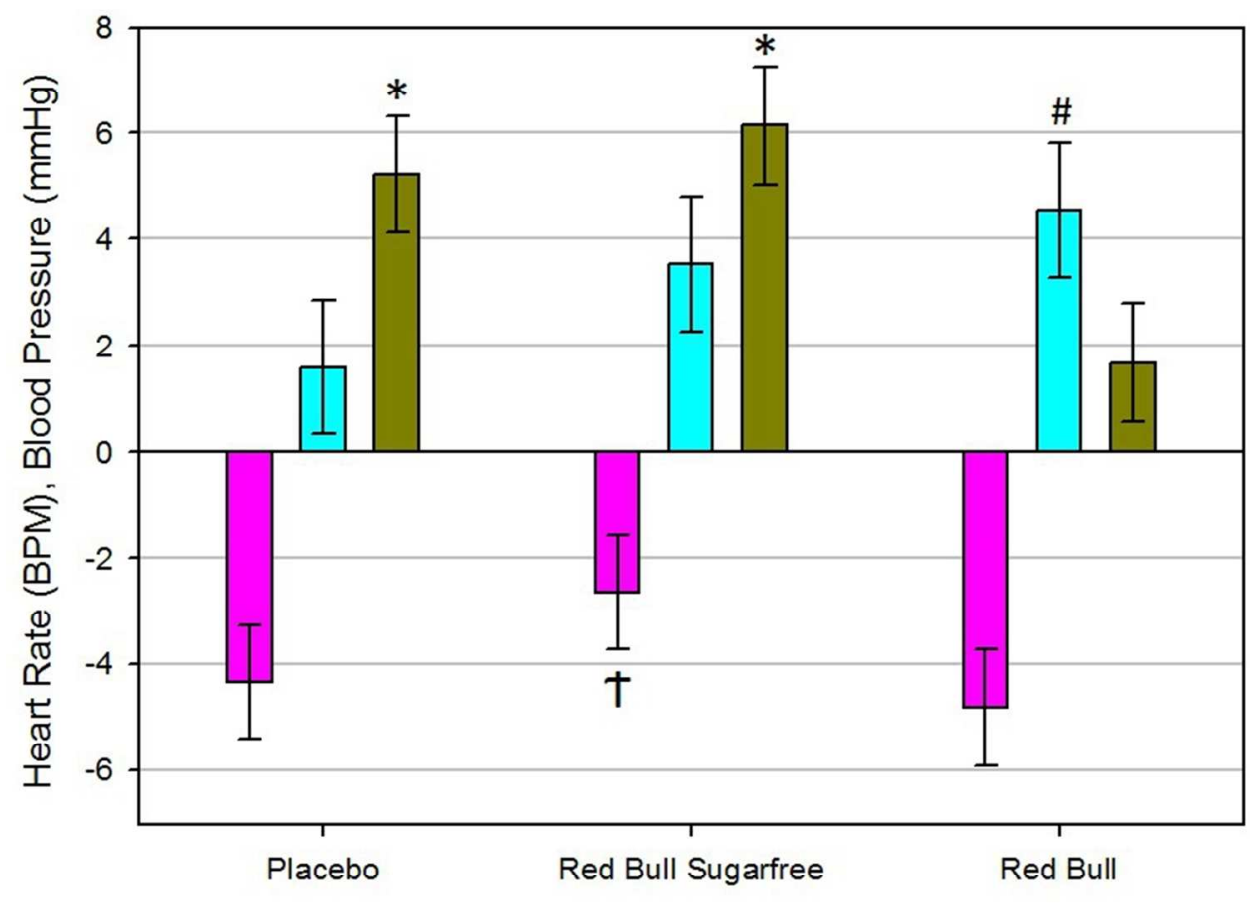

$\square$ Heart Rate

$\square$ Systolic Blood Pressure

$\square$ Diastolic Blood Pressure

$147 \times 132 \mathrm{~mm}(150 \times 150 \mathrm{DPI})$

http://mc.manuscriptcentral.com/jop 